$9 / 28 / 2010$

K.Yonehara

\title{
Proposal for High Pressure RF Cavity Test in the MTA
}

\section{Introduction}

In order to demonstrate the feasibility of high pressure hydrogen gas filled RF (HPRF) cavities for muon ionization cooling, an HPRF cavity must be tested with a high intensity charged beam. When an HPRF cavity is irradiatied with an intense beam each incident particle generates about 1000 electrons and ions per cubic centimeter in a high pressure cavity via ionization. These ionization electrons are influenced by the RF field and the RF quality factor goes down. This $\mathrm{Q}$ factor reduction will be a problem with a multi bunch beam, e.g., a muon beam for a muon collider consists of a 12 to 20 bunch train beam with 5 ns timing gap. Thus, the RF field must recover in few nano seconds. We propose to use a $400 \mathrm{MeV}$ proton beam in the MTA and measure a beam loading effect in the HPRF cavity and study the recovery mechanism of the RF field.

\section{Measurement}

The plot on the left side of Figure 1 shows the expected beam loading effect in the HPRF cavity with the MTA proton beam. First, the RF field turns on in $10 \mu \mathrm{s}$ without beam. The ramping time of the RF field is only determined by the RF $\mathrm{Q}$ factor. Once the beam is injected into the cavity at $\mathrm{t}=0 \mu \mathrm{s}$, the RF amplitude goes down immediately. This indicates the beam loading effect, i.e., the RF Q factor is reduced due to the development of a plasma in the cavity. The RF amplitude will reach an equilibrium when the ionization rate is equal to the recombination and thermal diffusion rates. Finally, the RF field recovers when the beam is off. The recovery time constant involves only the recombination and thermal diffusion processes.

The RF field recovery time can be shortened by doping the hygrogen gas with an electronegative gas. The plot on the right side of Figure 1 shows the effect of an electronegative dopant gas. A small amount of $\mathrm{SF}_{6}$ doping can yield a significantly fast $\mathrm{RF}$ field recovery time. $\mathrm{SF}_{6}$ was chosen in this simulation for convenience, but it is not a practical solution since the HPRF cavity will be operated at low temperature. . $\mathrm{N}_{2}$ gas will be used as a dopant gas since $\mathrm{NH}_{3}$ is immediately formed and can capture more electrons.

Hydrogen plasma dynamics will be observed by using a fast spectroscopic measurement. Dense hydrogen plasma emits thermal radiation light and a broadened hydrogen alpha line, as shown in Figure 2. The plasma density and temperature will be determined from analysis of spectroscopic light.

\section{Experimental setup}


Figure 3 shows a layout of the experimental setup. The HPRF is located in the MTA solenoid magnet. An RF pickup probe is used to observe the RF amplitude and frequency. Two probes were used in past measurement; one is an electric field sense antenna and the other is a magnetic field sense loop. There are two directional couplers to monitor the forward and reflected RF amplitudes. There is a circulator to eliminate RF reflection due to mismatching the resonance condition in a new waveguide configuration. Thus, one of directional couplers is inserted between the cavity and the circulator to monitor RF reflection.

There are six optical feedthroughs. Three of them are located on the top plate and the other three are on the side wall. There are three by two fiber sets. One fiber in each of the two sets is connected to a fast optical detector to measure white light, one is connected to a spectrometer, and the third will be available for future optional studies. In a previous test, we painted a wavelength shifter (TPB) at the end of the fiber to be able to observe UV light. We will repeat the same test in future experiments.

The beam intensity will be adjusted by varying the beam pulse width ( 5 to $20 \mu \mathrm{s}$ ). By putting a collimator in front of the cavity and tuning the focusing strength of final quadrupole lens, the beam intensity can be reduced a factor of 10 . Thus, the beam intensity can be varied from $10^{7}$ to $10^{9}$ protons/bunch. The beam intensity will be monitored by two toroid coils. One is located at downstream end of the final triplet and other is between downstream end of the beam collimator and the front of the cavity. A beam trigger will be generated by a beam counter. It detects reflected protons from a vacuum window. The beam is stopped in a beam absorber that is just downstream of the HPRF cavity.

An RF trigger signal will be provided from the MCR. This signal activates the Cmagnet to send Linac beam to the MTA beam line and also triggers the $805 \mathrm{MHz}$ Klystron for the HPRF cavity. The coincidence from beam counter and the RF trigger signal generates a trigger for the data acquisition system as shown in Figure 4. Fast signals, e.g. $\mathrm{RF}$ pickups and optical signals will be fed into a fast digital oscilloscope and slow signals, e.g. beam intensity and RF forward and reflected signals will go into the slow logic circuit and the slow digital oscilloscope, respectively.

\section{Safety issues}

There are two main safety assessments for this test. First, there is the hydrogen safety assessment. The hyrdrogen safety review has been completed and signed off. The most critical rule is that any energized device must be 15 feet away from the cavity. Second, there is the radiation shielding assessment. It specifies the limits on the beam intensity. We will be permitted to deliver a Linac pulse to the cavity at the rate of one per minute. In addition, the beam must be stopped in the MTA solenoid magnet to avoid beam spray issues in the MTA hall. To this end, the beam absorber is mounted downstream of the cavity. 


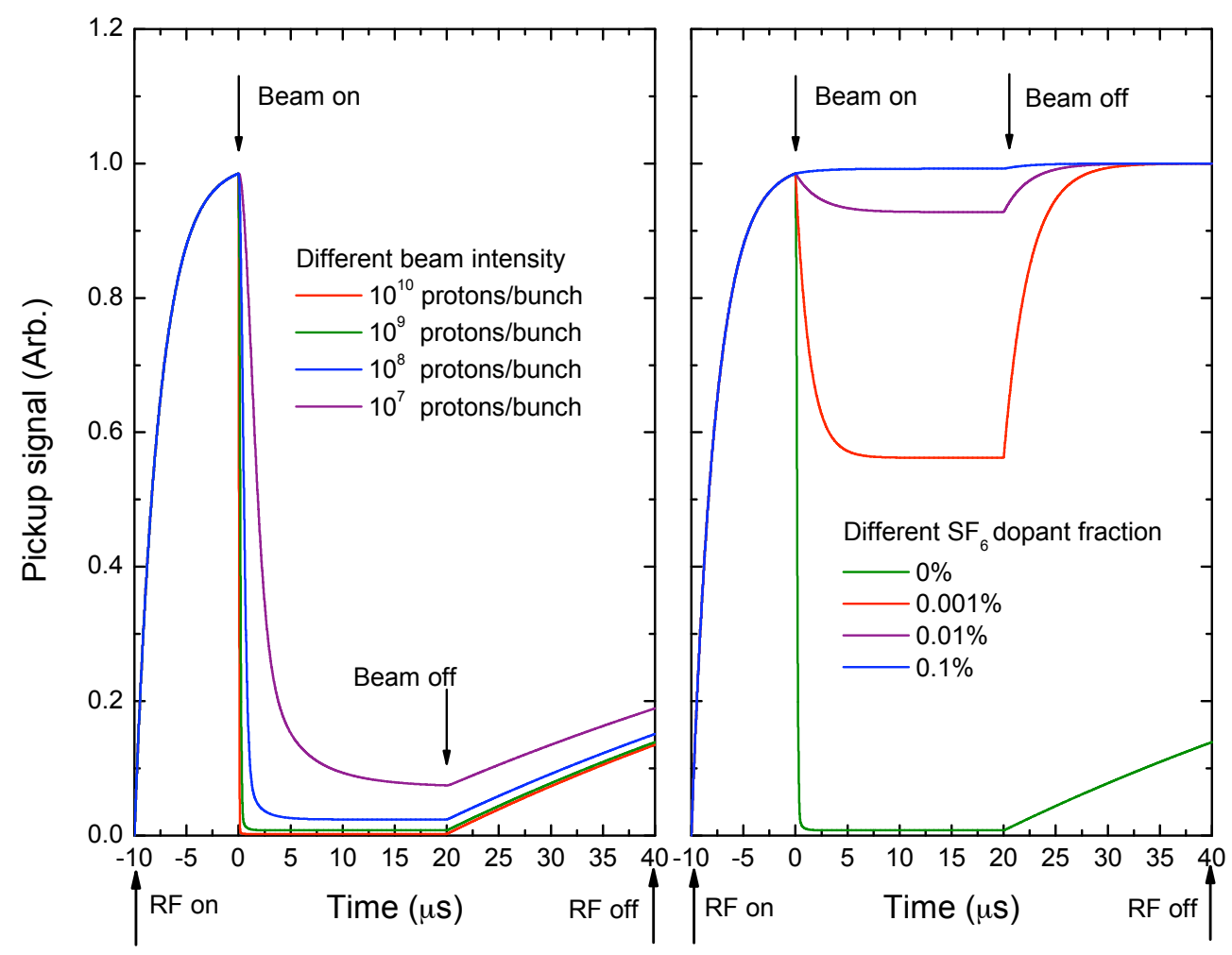

Figure 1: (Left) RF pickup signal of high pressure RF cavity. Hydrogen pressure is 1000 psi and recombination rate is $10^{-8} \mathrm{~cm}^{3} / \mathrm{s}$.

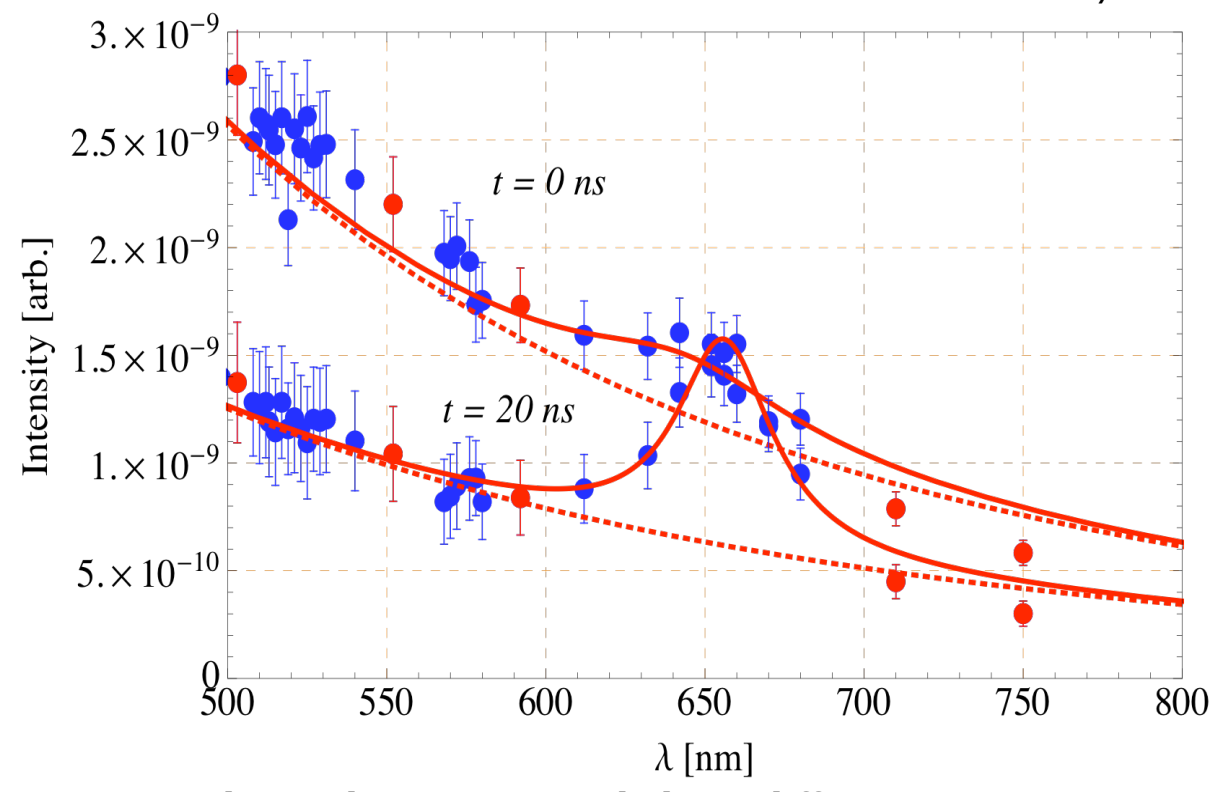

Figure 2: Observed spectroscopic light at different times. 0 ns is a peak intensity of emitted light from the cavity. The red points are the off resonance light and the blue ones are the resonance light, i.e. $656 \mathrm{~nm}$ is a hydrogen alpha line. There are also several copper lines around $510 \mathrm{~nm}$ and $550 \mathrm{~nm}$, respectively. The broken line is a fitting curve based on thermal 
radiation and solid one is a Lorentz fitting curve to extract the width of the broadened resonance light.

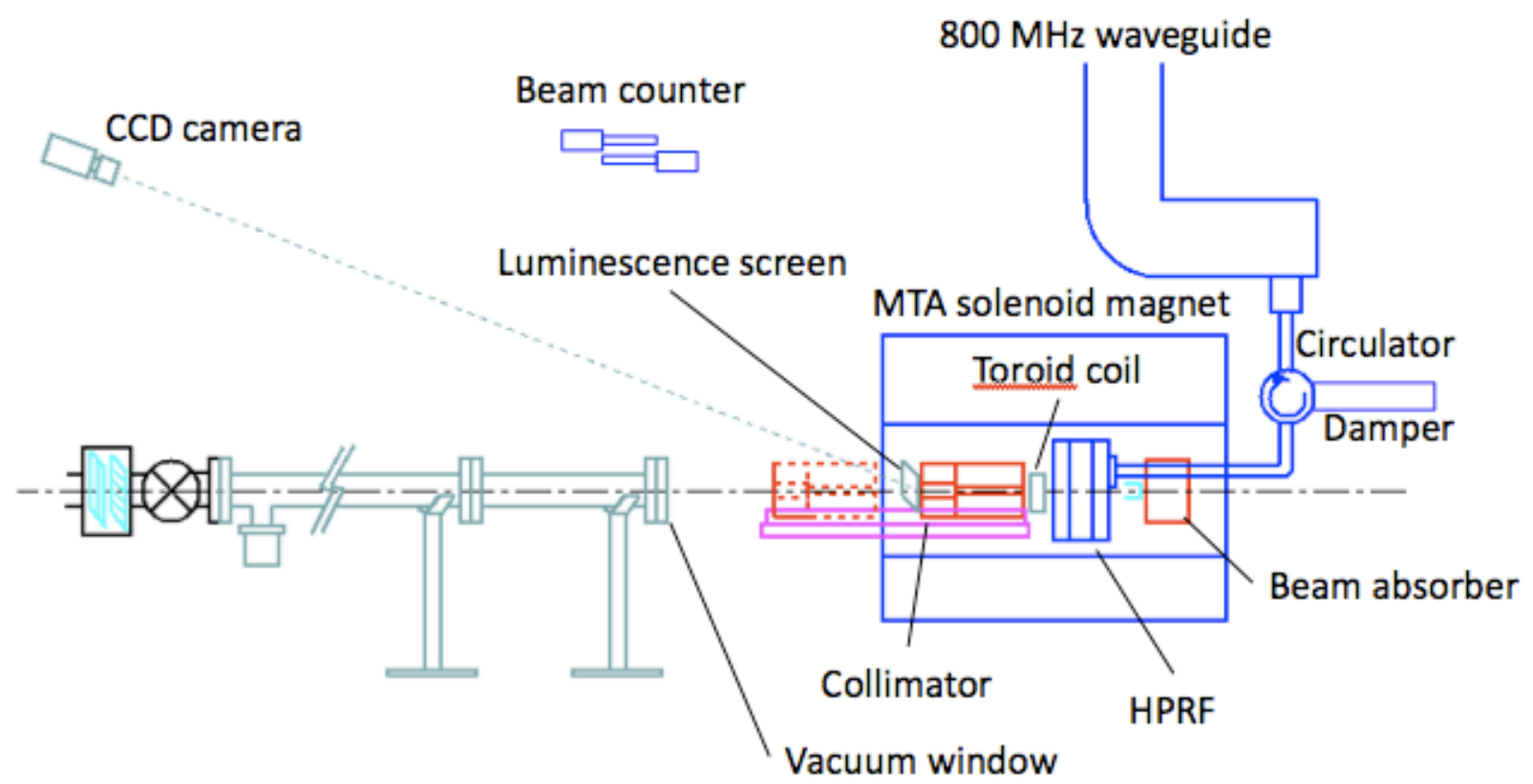

Figure 3: Layout of experimental devices.

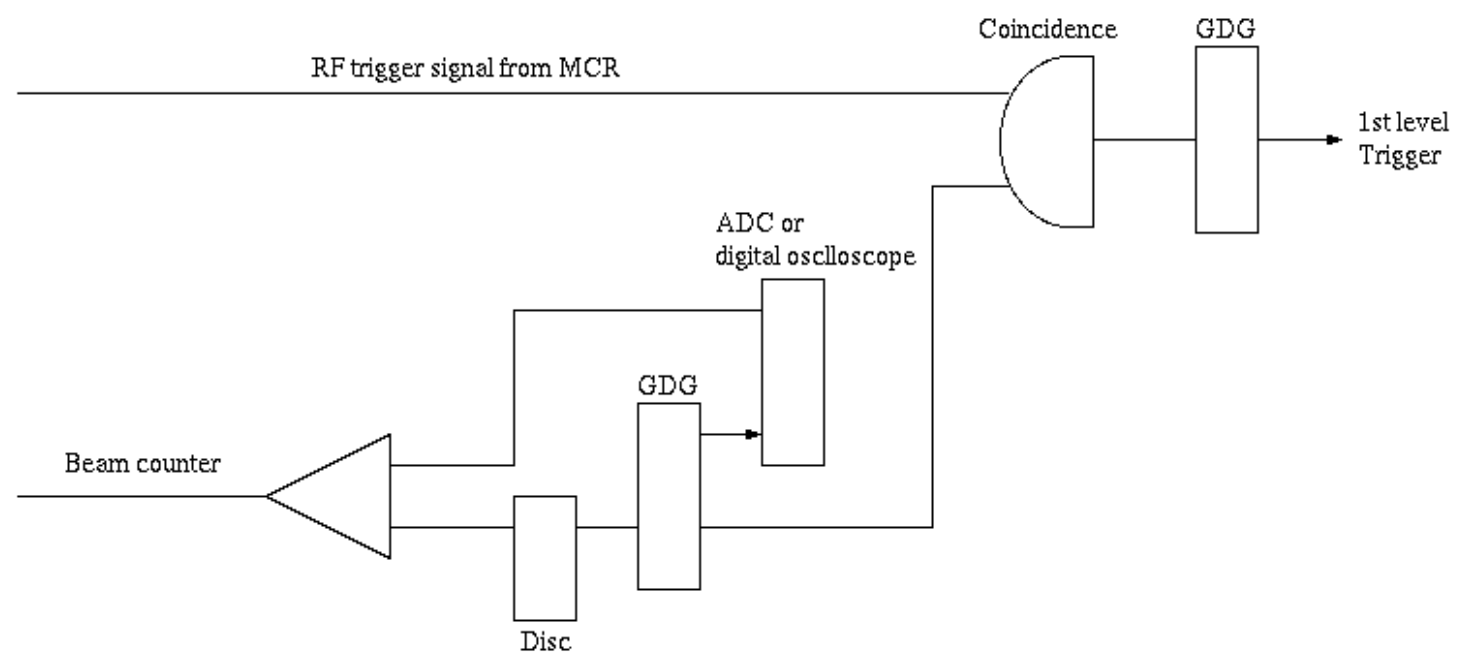

Figure 4: Trigger circuit.

\section{Criterion for down selection}

The RF field must recover in a few nano seconds the HPRF cavity concept is to be applicable for muon cooling channels. We would like to test some of the following issues in this experiment. 1) Measure the Paschen curve in a high pressure $800 \mathrm{MHz}$ cavity and determine if the slope is the same slope as in a DC field measurement. This would indicate that there is no frequency dependence on the Paschen curve. We need another experiment to verify this assumption. 2) Hydrogen plasma dynamics is well-known in the dilute 
condition. Our beam loading model is based on these past experimental results. The model will be tested in this experiment to verify the hydrogen plasma dynamic in the dilute case. 3) Polyatomic hydrogen clusters will be formed at very short time $(\ll 1 \mathrm{~ns})$. The recombination channels via the polyatomic hydrogen clusters are not taken into account our simulation. If this process is predominant, the recombination rate will be very fast. This process will be tested in the experiment. 4) Even if the recombination in pure hydrogen is slow, electronegative dopant gases can help to recover the RF field. This effect will be tested in the experiment. 5) The lifetime of positive ions are unclear. A long lifetime for heavy ions may affect the RF field with multi-bunch beam operation. This effect will be tested in the experiment. 6) We also need to investigate the hydrogen safety analysis and try to apply it to the cavities that would be used in a practical channel. This is not part of this experiment.

\section{Run Plan}

The entire HPRF running time will be 2.5 months including the preparation phase as shown in Figure 5. The start is strongly depending on the status of radiation shielding assessment. We are waiting for permission from the radiation safety committee. In first phase, we will have the HPRF cavity test without beam. It is a dry run for the beam test. We will take RF calibration data including RF $\mathrm{Q}$ factor and RF breakdown measurements with various gas conditions. We will also test the data acquisition system in this stage. In the second stage, we will measure the beam loading effect.

\section{Time table of HPRF test}

Total run duration will be 2.5 months

\section{\begin{tabular}{l|l|l} 
Sep & Oct & Nov
\end{tabular}}

RF modification, assemble

$\&$ calibration

Wave Guide \& Circulator

Beam line

Detector \& DAQ

Timing Calibration

$\mathrm{RF}$ test without beam

$\mathrm{RF}$ beam test
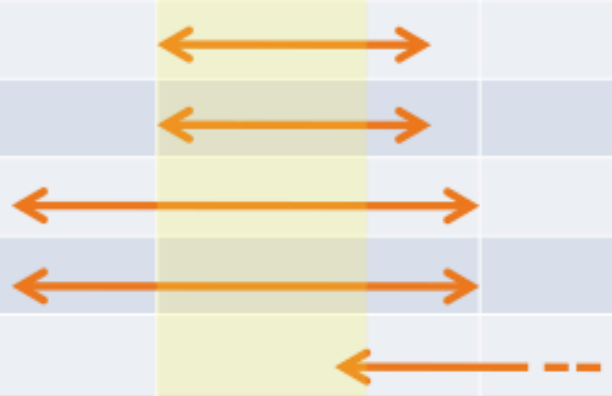

Figure 5: Time table of entire HPRF test. 


\section{Crew}

Katsuya Yonehara, Chief of experiment

Al Moretti, Co-chief of experiment

Milorad Popovic

Rolland Johnson

Gene Flanagan

Pierrick Hanlet

Ajit Kurup

Masahiro Notani

Moses Chung

Alvin Tollestrup

Jim Maloney

Ben Freemire

Giulia Collura 